\title{
Cysteine protease inhibition prevents mitochondrial apoptosis-inducing factor (AIF) release
}

\author{
Cell Death and Differentiation (2005) 12, 1445-1448. doi:10.1038/sj.cdd.4401687; published online 3 June 2005
}

Dear Editor,

During the last years, research on the molecular mechanisms governing caspase-dependent and -independent cell death has yielded a significant quantity of exciting works. As part of those efforts, apoptosis-inducing factor (AIF) was the first identified mitochondrial protein involved in caspase-independent cell death. ${ }^{1}$ Under physiological conditions, AIF is a mitochondrial FAD-dependent oxidoreductase that plays a role in oxidative phosphorylation. ${ }^{2}$ However, after a cellular insult, AIF is released from mitochondria and translocates to cytosol and nucleus where it achieves its proapoptotic function. ${ }^{1,3}$ Importantly, AIF seems a key factor in neuronal cell death ${ }^{4}$ and is involved in the early stages of development. ${ }^{5}$ Although alternative studies demonstrate that AIF could also be released in a caspase-dependent manner, ${ }^{6,7}$ AIF has been generally implicated in the caspase-independent mode of cell death. In this context, two recent papers from Otera et al. ${ }^{8}$ and Uren et al. ${ }^{9}$ demonstrated that AIF is a membrane-integrated protein that needs to be cleaved for becoming a soluble and apoptogenic protein. In fact, Otera et $a l^{8}$ states that AIF is released from mitochondria by a twostep process. First, AIF is cleaved in the mitochondrial matrix by the mitochondrial processing peptidase to form an innermembrane-anchored form. In a second step, AIF is processed, by an unidentified protease, in the intermembrane space of mitochondria (IMS) to yield a soluble and proapoptotic protein released to cytosol. But how is AIF cleaved in the IMS? Is it by a caspase? Apparently not. A third study from Polster et al. ${ }^{10}$ reported that after incubation of isolated mitochondria with different amounts of $\mathrm{Ca}^{2+}$, mitochondrial AIF release occurs through an N-terminal cleavage mediated by the $\mathrm{Ca}^{2+}$-dependent protease calpain I (also called $\mu$-calpain). These data provide a critical clue to understanding the regulation of AIF action. However, we here demonstrate that the mechanism governing AIF cleavage in the mitochondrial IMS is more complex than initially expected.

We first separate mitochondria from other organelles and debris on a discontinuous density gradient. The use of specific antibodies against AIF, LAMP1, and ERK, three markers of the mitochondrial, lysosomal, or cytoplasmic compartments, further confirmed the mitochondrial enrichment of our preparation. In fact, and as shown in Figure 1a, mitochondria are greatly enriched after Percoll gradient (AG) and the lysosomal and cytoplasmic cellular compartments are eliminated or reduced to a large extent. To address the underlying mechanisms by which AIF is cleaved into the IMS and released from mitochondria to induce death, we first determined by Edman microsequencing the $\mathrm{N}$-terminal amino acids of the two forms of AIF: the inner-membrane-anchored and the soluble and apoptogenic form. Our working hypothesis is that the protease responsible for the AIF cleavage must specifically yield the form released from mitochondria. In this way, the mouse anchored AIF presents an $\mathrm{N}$-terminal sequence starting at the amino acid A54 of the AIF precursor. On the other hand, when treating mitochondria in a calciumdepleted medium with atractyloside (Atr), an agent that induces mitochondrial AIF liberation, ${ }^{1,11,12}$ the protein became soluble and showed a lower apparent molecular weight (Figure 1b). This form, which we called tAIF, reveals an $\mathrm{N}$-terminal sequence starting at the amino acid L103 of the AIF precursor (indicating a proteolytic processing at position G102/L103). Intriguingly, like Atr, $\mathrm{Ca}^{2+}$ treatment induced in a dose-dependent manner an AIF proteolytic processing that yields the same soluble form of AIF: tAIF (Figure 1b). These results indicate that it is possible to induce AIF cleavage in the IMS in a $\mathrm{Ca}^{2+}$-dependent or in $\mathrm{Ca}^{2+}$-independent manner. Our results also implicate that proteases that are different from the previously identified $\mu$-calpain ${ }^{10}$ (which works exclusively in a $\mathrm{Ca}^{2+}$ environment) are implicated in AIF processing. Interestingly enough, the $\mathrm{N}$-terminal sequence of tAIF, obtained in our mitochondrial in vitro assays, is similar to the soluble form of AIF purified from cytosols of HeLa cells treated with etoposide, camptothecin, cisplatin, staurosporine, and MNNG (data not shown). This fact indicates that tAIF is the apoptogenic form of the protein released from mitochondria to cytosol after an apoptotic stimulus.

Using a fluorometric assessment, we next looked for a proteolytic activity provoking the $\mathrm{Ca}^{2+}$-independent AIF cleavage. As expected, in the absence of $\mathrm{Ca}^{2+}$ no relevant $\mu$-calpain activity was visualized (Figure 1c). Thus, we searched for other proteolytic activities that could regulate the Atr-dependent mitochondrial AIF cleavage. As shown in Figure 1c, supernatants from Atr-treated mitochondria contained a panel of proteases able to process z-Arg-Arg-amc (zRR), H-Arg-amc (R), and z-Phe-Arg-amc (zFR), three wellknown substrates used for the measurement of cathepsin $B$, cathepsin $\mathrm{H}$, and cathepsin L/S activity, respectively. In contrast, no caspase-3 activity (measured with Ac-Asp-GluVal-Asp-afc) was found (Figure 1c). Similar protease activities were measured in the supernatant obtained after the addition of digitonin to mitochondria (data not shown). These results 
a

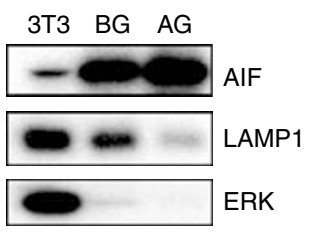

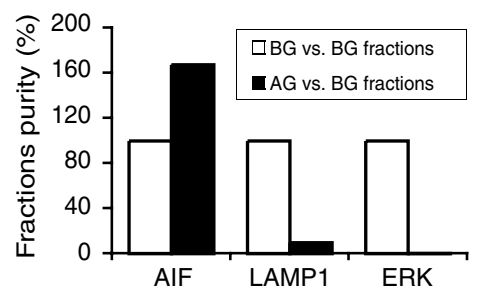

b

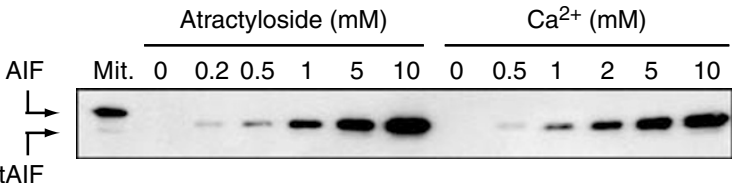

C

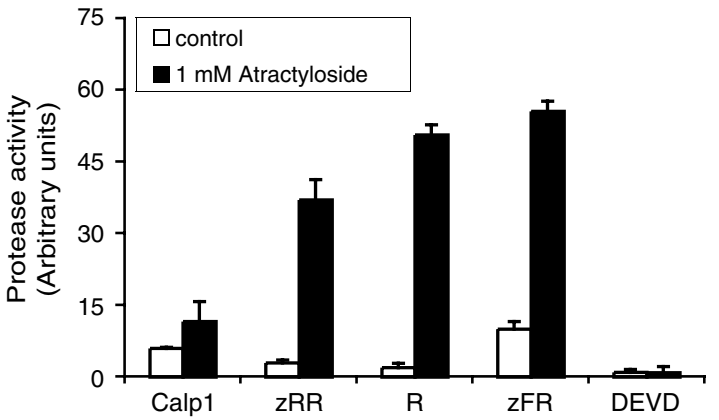

d

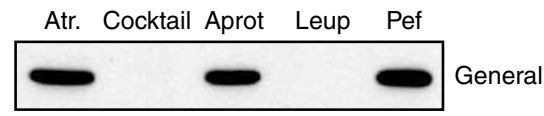

e

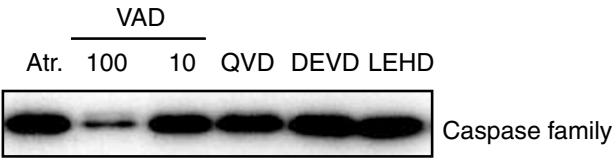

f

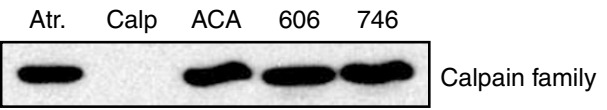

$\mathbf{g}$

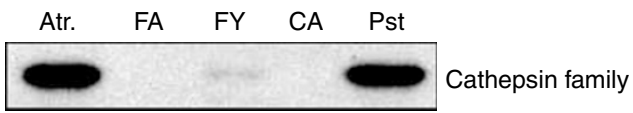

h

\begin{tabular}{lllll} 
Atr. & 132 & 115 & Epox Lact \\
\hline & & & \\
\hline & & &
\end{tabular}

i

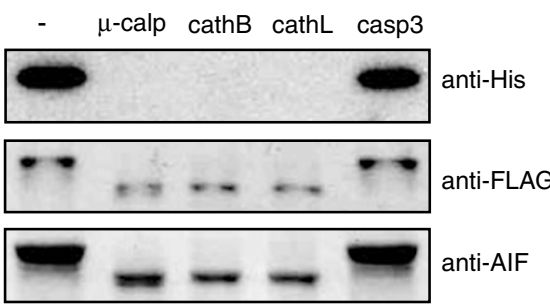

Figure 1 AIF is cleaved in the IMS of mitochondria by a noncaspase cysteine protease. (a) Mitochondria were purified in a Percoll ${ }^{\mathbb{R}}$ gradient as described in Table 1 and the assessment of the mitochondrial enrichment was performed by immunoblotting. Samples before and after Percoll gradient (BG and AG, respectively) were collected and subjected to a Western blot analysis of the mitochondrial AIF protein, the lysosomal LAMP1 marker, and the ERK cytosolic protein (left panel). Total protein extract from the mouse cell line NIH-3T3 were loaded as a control. The relative amount of each protein marker was quantified with the Image $J$ software and plotted as a percentage. Each histogram indicates the mitochondrial (AIF), lysosomal (LAMP1) or cytosolic (ERK) compartment represented in the final AG fraction, relative to the initial BG fraction (considered as a 100\%). Note that mitochondria were enriched after Percoll ${ }^{\mathbb{R}}$ gradient and, in contrast, the lysosomal and cytosolic fractions were greatly diminished or eliminated. (b) Atr. and $\mathrm{Ca}^{2+}$ treatments induce a dose-dependent mitochondrial AIF release. Soluble fractions recovered after Atr. or $\mathrm{Ca}^{2+}$ treatments were blotted for AIF detection. After the indicated treatment, and compared with untreated isolated mitochondria (Mit.), AlF exhibits a lower molecular weight. We named this new form of the protein tAIF. See text for details. (c) Assessment of the proteolytic activities present in the soluble fraction of Atr.-treated mitochondria. Calpain-1 (also called $\mu$-calpain), cathepsins B, H, L, and caspase-3 activities were determined using fluorescent probes from Calbiochem in supernatants from mitochondria left untreated (white bars) or treated with $1 \mathrm{mM}$ Atr. (black bars) for $35 \mathrm{~min}$ at $25^{\circ} \mathrm{C}$ as described in Table 1. Calp1: calpain-1 substrate; zRR: cathepsin B substrate; R: cathepsin H substrate; zFR: cathepsin L/S substrate; and DEVD: caspase-3 substrate. All reactions were performed at $25^{\circ} \mathrm{C}$ for $3 \mathrm{~h}$ and recorded in a Fluorimeter (Fluoroskan Ascent ${ }^{\mathrm{TM}}$, THERMO Labsystems). Protease activity is represented as fluorescence fold increase relative to an internal calibrator (unspecific substrate cleavage). Data are plotted as the means $( \pm$ S.D.) of at least five independent experiments. (d-h) Soluble fractions obtained from mitochondria treated with Atr. alone $(5 \mathrm{mM})$ or preincubated with a protease inhibitor cocktail from Roche $(\mathbf{d})$, or different caspase $(\mathbf{e})$, calpain $(\mathbf{f})$, cathepsin $(\mathbf{g})$, or proteasome $(\mathbf{h})$ inhibitors before Atr. treatment were blotted for AIF immunodetection as in (a). In panel d: Atr., atractyloside; Cocktail, Complete ${ }^{\mathbb{R}}$ Protease Inhibitor Cocktail (final concentration: $1 \times$ ); Aprot, aprotinin (100 $\mu \mathrm{M})$; Leup, leupeptin (250 nM); Pef, Pefabloc ${ }^{\mathbb{R}}$ SC (AEBSF) $(100 \mu \mathrm{M})$. In panel e: Atr., atractyloside; VAD, z-VAD.fmk (100-10 $\mu$ M); QVD, Q-VDOPh (100 $\mu \mathrm{M})$; DEVD, z-DEVD.fmk (100 $\mu \mathrm{M})$; LEHD, z-LEHD.fmk (100 $\mu \mathrm{M})$. In panel f: Atr., atractyloside; Calp, calpeptin (250 nM); ACA, N-acetyl-calpastatin peptide $(100 \mu \mathrm{M}) ;$; 606, PD $150606(100 \mu \mathrm{M}) ; 746$, PD $151746(100 \mu \mathrm{M})$. In panel g: Atr., atractyloside; FA, z-FA.fmk (250 nM); FY, z-FY.fmk (1 $\mu \mathrm{M}) ;$ CA, CA-074 (100 $\mu \mathrm{M}) ;$ Pst, pepstatin A (100 $\mu \mathrm{M})$. In panel h: Atr., atractyloside; 132, MG132 (250 nM); 115, MG115 (1 $\mu \mathrm{M})$; Epox, epoxomicin (100 $\mu \mathrm{M})$; Lact, lactacystin (100 $\mu \mathrm{M})$. All inhibitors were from Calbiochem, ICN, or Sigma. (i) Calpain and cathepsins cause AIF cleavage. Recombinant N-terminal His/C-terminal FLAG-tagged mouse AIF were produced with the help of the Pasteur Institute recombinant protein production platform. Briefly, mature mouse AlF $^{1}$ was produced from a Novagen pET28b expression vector and purified from Escherichia coli extracts on a nickel-NTA affinity matrix and stored in $50 \mathrm{mM} \mathrm{HEPES}, \mathrm{pH} 7.9,100 \mathrm{mM} \mathrm{NaCl}, 1 \mathrm{mM} \mathrm{DTT}$, and $10 \%$ glycerol. Recombinant AIF $(1 \mu \mathrm{g})$ was mixed with recombinant $\mu$-calpain $\left(\mu\right.$-calp) $\left(5 \mathrm{U} / \mathrm{ml}\right.$, Calbiochem) plus $2 \mathrm{mM} \mathrm{CaCl}_{2}$ or with cathepsin B (cathB) $(1 \mathrm{U} / \mathrm{ml}$, Calbiochem), cathepsin L (cathL) $(1 \mathrm{U} /$ $\mathrm{ml}$, Calbiochem), or caspase-3 (casp3) $\left(5 \mathrm{U} / \mathrm{ml}\right.$, Sigma). After $1 \mathrm{~h}$ at $37^{\circ} \mathrm{C}$, samples were boiled in SDS-PAGE sample buffer for 5 min and loaded onto a $10 \%$ SDSPAGE. After PVDF blotting, the AIF proteolytic processing was assessed by His, FLAG, or AIF immunodetection. Note that only the anti-FLAG antibody (Sigma), but not the anti-His antibody (Invitrogen), recognizes both unprocessed and processed AIF, indicating that the calpain- and cathepsin-mediated AIF cleavage occurs in the $\mathrm{N}$-terminal part of the protein.

indicate that the soluble fraction obtained after Atr or digitonin treatment contains a panel of proteases that are susceptible to regulate the $\mathrm{Ca}^{2+}$-independent AIF processing.

Taking into account the above results, we tested the effect of a broad panel of general or specific protease inhibitors on the Atr-mediated AIF release (Table 1). Among the general protease inhibitors tested, only the cysteine protease blocking agents chymostatin, E64, and leupeptin completely precluded AIF cleavage even at lower concentrations $(1 \mu \mathrm{M}$ for chymostatin and E64 and $250 \mathrm{nM}$ for leupeptin) (Table 1). 
Table 1 Effect of protease inhibitors on AIF release

\begin{tabular}{|c|c|c|c|c|c|c|c|c|c|c|c|}
\hline & $100 \mu \mathrm{M}$ & $10 \mu \mathrm{M}$ & $1 \mu \mathrm{M}$ & $0.5 \mu \mathrm{M}$ & $0.25 \mu \mathrm{M}$ & & $100 \mu \mathrm{M}$ & $10 \mu \mathrm{M}$ & $1 \mu \mathrm{M}$ & $0.5 \mu \mathrm{M}$ & $0.25 \mu \mathrm{M}$ \\
\hline General inhibitors & & & & & & Calpain inhibitors & & & & & \\
\hline Aminopeptidases & & & & & & MG-101 & + & + & + & + & + \\
\hline Amastatin & - & & & & & Ac-LLM-CHO & + & + & + & - & - \\
\hline Aspartic proteases & & & & & & Calpeptin & + & + & + & + & + \\
\hline Pepstatin A & - & - & & & & Calpastatin peptide & + & - & & & \\
\hline Cysteine proteases & & & & & & PD 150606 & + & - & & & \\
\hline E-64 & + & + & + & - & & PD 151746 & + & - & & & \\
\hline Leupeptin & + & + & + & + & + & Caspase inhibitors & & & & & \\
\hline Chymostatin & + & + & + & - & & Boc-D.fmk & - & - & & & \\
\hline Metalloproteases & & & & & & z-VAD.fmk & $+/-$ & - & & & \\
\hline BAPTA-AM & - & - & & & & QVD-OPh & - & - & & & \\
\hline Bestatin & - & - & & & & z-VDAVD.fmk & - & - & & & \\
\hline GM6001 & - & - & & & & z-DEVD.fmk & - & - & & & \\
\hline O-phenanthroline & _- & - & & & & z-VEID.fmk & - & - & & & \\
\hline Serine proteases & & & & & & z-IETD.fmk & - & - & & & \\
\hline 3,4-DCl & - & - & & & & z-LEHD.fmk & - & - & & & \\
\hline Aprotinin & - & - & & & & Cathepsin inhibitors & & & & & \\
\hline Pefabloc (AEBSF) & - & - & & & & z-FA.fmk & + & + & + & + & + \\
\hline PMSF & - & - & & & & z-FY.fmk & + & + & + & - & - \\
\hline TLCK & + & - & & & & Pepstatin A & - & - & & & \\
\hline \multirow[t]{8}{*}{ TPCK } & - & - & & & & $\mathrm{CA}-074$ & + & $+1-$ & - & & \\
\hline & & & & & & Proteasome inhibitors & & & & & \\
\hline & & & & & & z-LLF-CHO & - & - & & & \\
\hline & & & & & & Epoxomicin & - & - & & & \\
\hline & & & & & & Lactacystin & - & - & & & \\
\hline & & & & & & MG-115 & + & + & + & - & - \\
\hline & & & & & & MG-132 & + & + & + & + & + \\
\hline & & & & $1 \times$ & & NLVS & + & + & + & - & - \\
\hline Protease inhibitor cocktail & & & & & & & & & & & \\
\hline (aprotinin, leupeptin and pefabloc) & & & & + & & & & & & & \\
\hline
\end{tabular}

$\mathrm{BALB} / \mathrm{c}$ mouse liver mitochondria were isolated as previously described ${ }^{1}$ and resuspended in a buffer containing $10 \mathrm{mM} \mathrm{Hepes/KOH} \mathrm{pH} 7.2,250 \mathrm{mM}$ sucrose, and $5 \mathrm{mM}$ EGTA. Mitochondrial protein concentration was determined by the Bio-Rad DC Protein Assay ${ }^{\mathbb{R}}$ method. $300 \mu \mathrm{g}$ of mitochondria were pre-treated for 15 min at $4{ }^{\circ} \mathrm{C}$ with different concentrations of the indicated protease inhibitor before addition of Atractyloside (Atr., $5 \mathrm{mM}$ ). The mixture was incubated for 35 min at $20^{\circ} \mathrm{C}$ and centrifuged $\left(30 \mathrm{~min}, 4^{\circ} \mathrm{C}, 13,400 \mathrm{~g}\right)$ to obtain the supernatant containing proteins released from mitochondria. Supernatants were further re-clarified $\left(90\right.$ min, $4^{\circ} \mathrm{C}$, $100000 \mathrm{~g}$ ) to produce a soluble fraction. Equal volumes of these fractions were loaded onto a 12\% SDS-polyacrylamide gel. After blotting, PVDF filters (Millipore, Bedford, MA) were probed with an anti-AIF antibody directed against the C-terminal part of the protein (Sigma-Aldrich, Saint-Quentin Fallavier, France) and detected by an anti-rabbit IgG HRP conjugated antibody (Sigma) according to standard procedures. Table compiles the results obtained after visual immunoblot analysis, which are represented as +: inhibition of AIF cleavage and release (= absence of cleaved AIF in the soluble fraction), or -: no inhibition of the Atr-mediated AIF cleavage and release ( = presence of cleaved AIF in the soluble fraction). Each inhibitor and concentration has been tested at least three times, yielding comparable results. All inhibitors were from Calbiochem, ICN, or Sigma

Unexpectedly, a protease cocktail widely used in the analysis of the release of proapoptotic proteins from mitochondria successfully inhibited Atr-induced AIF cleavage and release (Table 1 and Figure 1d). This could be explained by the inclusion of leupeptin into the cocktail inhibitors. By contrast, none of the inhibitors reported to preclude aminopeptidase, aspartic protease, metalloprotease or serine protease action showed any effect on mitochondrial AIF liberation (Table 1). Overall, our fluorometric and pharmacological data point towards an implication of cysteine proteases in the regulation of AIF cleavage.

Next, we investigated whether major families of cysteine proteases implicated in apoptosis (calpains, caspases, cathepsins, and proteasome) regulate mitochondrial AIF liberation. In fact, and as shown in Table 1, some of the calpain, cathepsin, or even proteasome inhibitors precluded AIF cleavage. Only caspases did not seem to be involved in the release of the protein (Table 1). However, when used at high doses ( $>100 \mu \mathrm{M})$, zVAD.fmk partially alleviates AIF cleavage and release (Table 1 and Figure 1e). This can be explained by the fact that, when used at these higher doses, zVAD.fmk is no longer specific and could also inhibit other cysteine proteases, like calpains ${ }^{13}$ or cathepsins. ${ }^{14,15}$ Within the panoply of other cysteine protease inhibitors tested, the most powerful agents precluding AIF release were: MG101 and calpeptin (calpain inhibitors); zFA.fmk (cathepsin inhibitor); and MG132 (an inhibitor of the proteasome). In a lesser degree, zFY.fmk, CA-074, Ac-LLM-CHO, MG115, and NLVS also inhibit AIF release (Table 1 and Figure $1 \mathrm{f}-\mathrm{h}$ ). Unfortunately, the use of a pharmacological approach presents a limit, which is protease specificity. Indeed, all reagents precluding the AIF mitochondrial processing (including proteasome inhibitors) inhibit two major families of cystein proteases: calpains and cathepsins. ${ }^{16-18}$ However, as calpains are inactive enzymes in the Atr calcium-depleted system (Figure 1c), our results point toward a cathepsin implication in $\mathrm{Ca}^{2+}$-independent AIF processing. Thus, we looked for an individual cathepsin protease integrating the results presented above.

Cathepsins are subdivided into three distinct groups: serine proteases (e.g. cathepsins $A$ and $G$ ), aspartate proteases (e.g. cathepsins $D$ and $E$ ) and cystein proteases (e.g. B, L, and $S) .{ }^{18}$ Intriguingly, only the cathepsin cystein proteases $B$, $\mathrm{L}$, and $\mathrm{S}$ cleaved mouse recombinant AIF (Figure 1i and data 
not shown). More importantly, this cleavage yields tAIF, the soluble form of AIF (N-terminal sequence starting at L103). Interestingly enough, in a calcium-supplemented context, the protease $\mu$-calpain also provokes an AIF processing that yields tAIF (Figure 1i). In contrast, neither cathepsin D (a protease implicated in caspase-independent apoptosis) ${ }^{19}$ nor caspase-2, -3, and -9 (three caspases localized in the mitochondrial intermembrane space) were able to process AIF (Figure 1i and data not shown). These results indicate that there is no one enzyme responsible for the $\mathrm{Ca}^{2+}$ independent AIF processing. In fact, the protein could be cleaved into tAIF by cathepsins $B, L$, and $S$, three cystein proteases present in the soluble fraction of Atr-treated mitochondria (Figure 1c).

Together with the results obtained from Polster et al., ${ }^{10}$ the present study reveals progress in the knowledge of AIF, one of the major agents implicated in caspase-independent apoptosis. Accepting the complexity of cell death regulation and execution, we could assume that there are different mechanisms that directly provoke the release of this protein from mitochondria, implicating the cathepsins $B, L$, and $S$ and/or $\mu$-calpain. Indeed, the protein could be released from mitochondria in a $\mathrm{Ca}^{2+} / \mu$-calpain-dependent ${ }^{10}$ or in $\mathrm{Ca}^{2+}$-independent/cathepsin-dependent manner. In any case, AIF is cleaved and released from mitochondria in a caspaseindependent manner. In a cellular context, and when caspases are placed upstream of lysosomes and mitochondria, our work supports the previously reported possibility that caspases increase lysosomal or mitochondrial permeabilization, thereby provoking cathepsin and/or calpain activation. ${ }^{18,20}$ This activation might cause further AIF cleavage and release. In this case, the sequence of cell death events describes a single-cell death pathway that includes both caspases and other caspase-independent effectors like cathepsins, calpains, or AIF. Anyhow, our results suggest that all trials on AIF release must be performed in the presence of low amounts of zVAD.fmk or with a protease inhibitor set without leupeptin. Importantly enough, AIF release must be evaluated with an antibody reacting against the C-terminal part of AIF, which is conserved after AIFmitochondrial processing (Figure 1i).

\section{Acknowledgements}

We thank Marika Sarfati and Luigi Ravagnan for critical comments and review of the manuscript. This work was supported by grants from the Association pour la Recherche sur le Cancer (Contract No. 4812), Fondation de France, and the Pasteur-Weizmann Scientific Council (to SAS). VJY was supported by a Marie Curie Intra-European fellowship within the 6th European Community Framework Programme (contract MEIF-2003-501887). CD and PS hold postdoctoral fellowships from Fondation de France and Fondation Manlio Cantarini, respectively. RSM and $\mathrm{MB}$ hold $\mathrm{PhD}$ fellowships from Fondation Hariri and MENRT, respectively.

\author{
VJ Yuste ${ }^{1}$, RS Moubarak ${ }^{1}$, C Delettre ${ }^{1}, M$ Bras $^{1}$, \\ $P$ Sancho $^{1}, N$ Robert $^{1}, J$ d'Alayer $^{2}$ and SA Susin ${ }^{*, 1}$ \\ ${ }^{1}$ Apoptose et Système Immunitaire, CNRS-URA 1961, Institut Pasteur 25 rue \\ du Dr Roux, 75015 Paris, France \\ 2 Plate-Forme d'Analyse et de Microséquençage des Protéines, Institut Pasteur, \\ 25 rue du Dr Roux, 75015 Paris, France \\ * Corresponding author: SA Susin, Institut Pasteur, 25, rue du Dr. Roux. \\ Tel: + 33-1-40-61-31-84; Fax: + 33-1-40-61-31-86; \\ E-mail: susin@pasteur.fr
}

1. Susin SA et al. (1999) Nature 397: 441-446

2. Vahsen $\mathrm{N}$ et al. (2004) EMBO J. 23: 4679-4689

3. Yu SW et al. (2002) Science 297: 259-263

4. Cheung EC et al. (2005) J. Neurosci. 25: 1324-1334

5. Joza N et al. (2001) Nature 410: 549-554

6. Arnoult D et al. (2003) Cell Death Differ. 10: 845-849

7. Arnoult $D$ et al. (2003) EMBO J. 22: 4385-4399

8. Otera $\mathrm{H}$ et al. (2005) EMBO J. 24: $1375-1386$

9. Uren RT et al. (2005) J. Biol. Chem. 280: 2266-2274

10. Polster BM et al. (2005) J. Biol. Chem. 280: 6447-6454

11. Zamzami N et al. (1996) FEBS Lett. 384: 53-57

12. Vancompernolle K et al. (1998) FEBS Lett. 438: 150-158

13. Wolf BB et al. (1999) Blood 94: 1683-1692

14. Schotte $P$ et al. (1999) FEBS Lett. 442: 117-121

15. Rozman-Pungercar J et al. (2003) Cell Death Differ. 10: 881-888

16. Sasaki T et al. (1990) J. Enzyme Inhibitors 3: 195-201

17. Lee DH and Goldberg AL (1998) Trends Cell Biol. 8: 397-403

18. Johnson DE (2000) Leukemia 14: 1695-1703

19. Bidere $\mathrm{N}$ et al. (2003) J. Biol. Chem. 278: 31401-31411

20. Foghsgaard L et al. (2001) J. Cell Biol. 153: 999-1010 